\title{
Nécrolyse épidermique toxique due à I'ipilimumab et au nivolumab chez une patiente souffrant d'un mélanome métastatique
}

\author{
par Marika Lepage-Légaré, Sandrine Léger, Hugo Ricignuolo, Gabrielle St-Louis et Thomas Joly-Mischlich
}

J. Can. Pharm. Hosp. 2021;74(3):294-7

DOI: 10.4212/cjhp.v74i3.3158

\section{INTRODUCTION}

Depuis quelques années, l'immunothérapie est utilisée dans le traitement antinéoplasique et son efficacité se démontre pour un nombre grandissant d'indications. Un de ses effets est dû à la stimulation du système immunitaire par l'inhibition des points de contrôle, ce qui permet l'activation des lymphocytes $\mathrm{T}$ afin qu'ils exercent leur effet cytotoxique sur les cellules tumorales ${ }^{1}$. Cette stimulation du système immunitaire est toutefois associée à de possibles réactions indésirables à médiation immunitaire et à un risque de réactivation de maladies auto-immunes ${ }^{1,2}$. Le cas présenté dans cet article décrit une réaction rapide de nécrolyse épidermique toxique (NET) associée à la prise d'ipilimumab (anti-CTLA4) et de nivolumab (anti-PD1) par une patiente souffrant d'un mélanome métastatique.

\section{DESCRIPTION DU CAS}

Il s'agit d'une femme de 58 ans, pesant $90 \mathrm{~kg}$, mesurant $158 \mathrm{~cm}$ et sans allergie médicamenteuse connue.* Elle souffre d'hypertension artérielle, de diabète de type II et d'hypothyroïdie. Elle a reçu un nouveau diagnostic de mélanome métastatique avec une mutation V600E sur le gène BRAF. Trois métastases cérébrales ont initialement causé deux épisodes convulsifs, ce pourquoi elle s'est vu prescrire du lévétiracétam. La patiente préférant la double immunothérapie à une simple immunothérapie ou à une thérapie anti-BRAF, elle entreprend un premier traitement constitué de quatre doses d'ipilumumab à raison de $3 \mathrm{mg} / \mathrm{kg}$ et de nivolumab à raison de $1 \mathrm{mg} / \mathrm{kg}$ toutes les trois semaines. Elle reçoit son premier cycle au jour 0 (J0).

${ }^{*}$ Le consentement écrit de la famille de la patiente a été obtenu pour publier ce rapport de cas et les images qui lui sont associées, conformément aux exigences du comité d’éthique de la recherche de létablissement concerné.
$\mathrm{Au} \mathrm{J6}$, elle se présente à l'urgence pour une éruption cutanée qui progresse rapidement, apparue dans son dos au J3. Au J5, elle présente un épisode de lipothymie sans conséquence significative. À lévaluation initiale, les lésions maculopapulaires érythémateuses sétendent sur plus de $30 \%$ de la surface corporelle (SC). La patiente reçoit un traitement de prednisone dosé à $90 \mathrm{mg}(1 \mathrm{mg} / \mathrm{kg} / \mathrm{jour})$, de diprosone topique à appliquer deux fois par jour et de $50 \mathrm{mg}$ de diphenhydramine intraveineux toutes les six heures au besoin. Elle a une créatinine sérique de base de $60 \mu \mathrm{mol} / \mathrm{L}$, mais elle présente une insuffisance rénale aiguë avec une créatinine sérique de $108 \mu \mathrm{mol} / \mathrm{L}$ au J6, résolue par un soluté de réhydratation. $\mathrm{Au} \mathrm{J}$, on note une augmentation légère de l'alanine aminotransférase (ALT) à 43 UI/L (valeur normale : 0-37 UI/L) comparativement à $22 \mathrm{UI} / \mathrm{L}$ une semaine avant l'immunothérapie. Ses autres résultats de laboratoire sont normaux. Une histoire médicamenteuse a été réalisée à l'arrivée de la patiente (tableau 1 ). Du J8 au J10, la réaction cutanée progresse jusqu'à atteindre 65 à $70 \%$ de sa SC et des phlyctènes apparaissent sur $20 \%$ de celle-ci. Le signe de Nikolsky est positif et les trois muqueuses sont atteintes. Son score de Scorten est estimé à $4 / 10$ et est associé à $58 \%$ de risque de décès ${ }^{3}$. Un dermatologue confirme la suspicion d'un syndrome de Stevens-Johnson (SSJ) évoluant vers une NET. Au J10, trois biopsies ont été réalisées et des photos de la réaction ont été prises (figure 1). Au J11, léruption cutanée couvre $90 \%$ de la SC et les phlyctènes, plus de $20 \%$. Puisque la réaction progresse rapidement, la patiente est transférée à l'unité des grands brûlés d'un centre hospitalier tertiaire, où elle reçoit des soins de plaie adaptés et de la prednisone à raison de $2 \mathrm{mg} / \mathrm{kg} / \mathrm{jour}$. Elle n’a pas reçu de cyclosporine, d'etanercept ni d'immunoglobulines. Lors de ce séjour, les lésions cutanées évoluent en cellulite multigermes traitée avec du meropenem pendant 11 jours.

Après une évolution favorable des lésions, au J32, la patiente est à nouveau transférée à son hôpital 
TABLEAU 1. Histoire médicamenteuse à l'admission

\begin{tabular}{|c|c|c|}
\hline Médicament & Posologie & Date \\
\hline Médication au jour $0^{\mathrm{a}}$ & & $\begin{array}{l}\text { Date de début } \\
\text { de la médicament }\end{array}$ \\
\hline Nivolumab & $\begin{array}{l}1 \mathrm{mg} / \mathrm{kg} \text { intraveineux tous les } 21 \text { jours pour } 4 \text { doses, puis } 3 \mathrm{mg} / \mathrm{kg} \\
\text { tous les } 14 \text { jours pour une durée indéterminée }\end{array}$ & Jour 0 \\
\hline Ipilimumab & $3 \mathrm{mg} / \mathrm{kg}$ intraveineux tous les 21 jours pour 4 doses & Jour 0 \\
\hline Dexlansoprazole & $60 \mathrm{mg}$ une fois par jour & Jour -77 \\
\hline $\begin{array}{l}\text { Tramadol/ } \\
\text { acétaminophène }\end{array}$ & $\begin{array}{l}37,5 \text { / } 325 \mathrm{mg} 1 \text { comprimé toutes les } 6 \text { heures au besoin } \\
\text { Prise réelle : au besoin }\end{array}$ & Jour -77 \\
\hline Amlodipine & $10 \mathrm{mg}$ une fois par jour & Jour -14 \\
\hline Calcium / vitamine D & $500 \mathrm{mg} / 400 \mathrm{UI}$ deux fois par jour & Plus de 2 mois \\
\hline Lévétiracétam & $500 \mathrm{mg}$ deux fois par jour & Jour -112 \\
\hline Lévothyroxine & $0,05 \mathrm{mg}$ une fois par jour & Jour -112 \\
\hline Metformine & $1000 \mathrm{mg}$ deux fois par jour & Jour -126 \\
\hline Saxagliptine & $5 \mathrm{mg}$ une fois par jour & Jour -126 \\
\hline Insuline NPH & 20 unités le matin et 12 unités au coucher & Jour -112 \\
\hline Insuline lispro & 10 unités le matin, le midi et au souper & Jour -112 \\
\hline Lorazépam & $\begin{array}{l}0,5 \mathrm{mg} \text { une fois par jour au besoin en cas d'insomnie } \\
\text { Prise réelle : au besoin }\end{array}$ & Jour -112 \\
\hline Crème hydratante & $\begin{array}{l}\text { Application sur la corne et la peau sèche des pieds et des mains deux fois } \\
\text { par jour au besoin } \\
\text { Prise réelle : au besoin }\end{array}$ & Jour -112 \\
\hline Changements récents de la médication & & $\begin{array}{l}\text { Date de fin } \\
\text { de la médicament }\end{array}$ \\
\hline Périndopril & $4 \mathrm{mg}$ une fois par jour & Jour -14 \\
\hline Labétalol & $100 \mathrm{mg}$ deux fois par jour & Jour -42 \\
\hline $\mathrm{NaCl}$ & $1 \mathrm{~g}$ trois fois par jour & Jour -42 \\
\hline Dexaméthasone & $3 \mathrm{mg}$ deux fois par jour & Jour -14 \\
\hline Triméthoprime-sulfaméthoxazole & $160 / 800$ mg trois fois par semaine & Jour -14 \\
\hline
\end{tabular}

ajour $0=$ premier cycle de la double immunothérapie.

${ }^{\mathrm{b}}$ Neutral protamine Hagedorn.
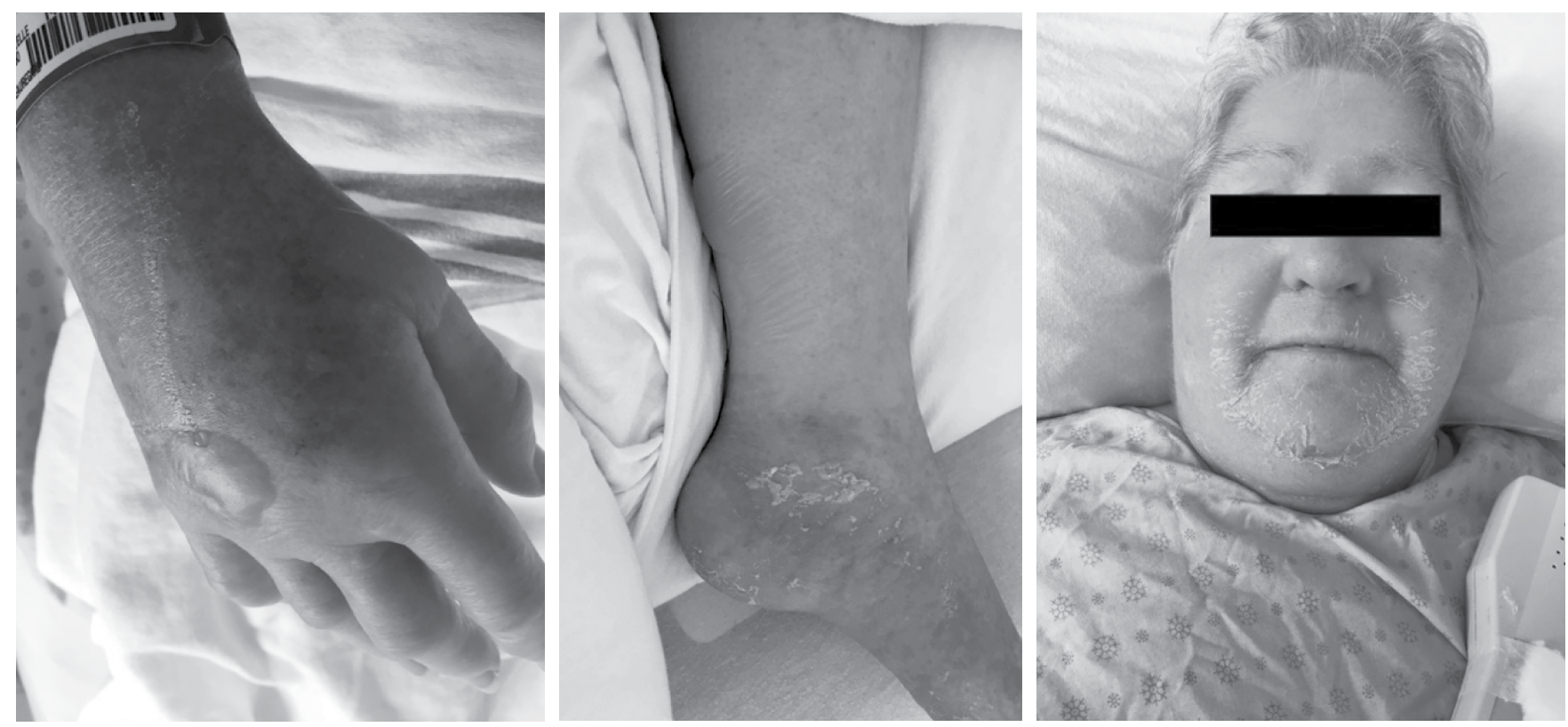

FIGURE 1. État de la réaction cutanée 10 jours après l'administration d'une première dose d'ipilimumab et de nivolumab. 
d'appartenance. Un débalancement de son diabète sous corticostéroïdes suivi en endocrinologie et une pneumonie à Pneumocystis jiroveci pour laquelle elle a reçu un traitement de triméthoprime-sulfaméthoxazole de 21 jours prolongent son hospitalisation. Le traitement a été bien toléré et elle a obtenu son congé de l'hôpital au J56. À son départ, elle a reçu une ordonnance de sevrage de prednisone et de suivi en dermatologie. La patiente a reçu de la prednisone pendant cinq mois.

Le résultat des biopsies démontre une nécrose épidermique presque complète avec un discret infiltrat dermique lymphohistiocytaire périvasculaire et les immunofluorescences directes sont négatives; le résultat tend donc à confirmer la présence d'une NET.

\section{DISCUSSION}

Plusieurs éléments ont mené au diagnostic de NET chez cette patiente. Le malaise qui l'a menée à consulter à l'urgence s'apparente au prodrome précédant une NET, et l'éruption érythémateuse qui a rapidement progressé en phlyctènes et en desquamation est caractéristique d'une réaction cutanée causée par un médicament. Par ailleurs, latteinte des trois muqueuses et le signe de Nikolsky positif orientaient aussi vers ce diagnostic confirmé par une biopsie. Un score de Naranjo de sept indique que la NET a probablement été causée par la combinaison d'ipilimumab et de nivolumab ${ }^{4}$ et c'est aussi ce qu'ont conclu les cliniciens.

Quelques rapports de cas de SSJ et de NET portant sur l'ipilimumab et le nivolumab sont présentés dans la littérature scientifique. Dans un premier cas, une femme de 64 ans traitée pour un mélanome métastatique réfractaire à l'ipilimumab a développé ces symptômes quatre semaines après le début d'un traitement au nivolumab ${ }^{5}$. Dans ce cas, l'ajout d'immunoglobulines et de cyclosporine à la cortisone à haute dose a permis de contenir la réaction. Dans un deuxième $\mathrm{cas}^{6}$, un homme de 62 ans, traité pour un mélanome métastatique, a développé une NET au jour 4 après le deuxième cycle de double immunothérapie, soit 25 jours après le début du traitement. Malgré la prise en charge du patient par l'ajout d'immunoglobulines et de cyclosporine, il est décédé d'une défaillance d’organes multiples. Un autre patient de 54 ans traité pour un lymphome folliculaire a développé une NET 10 jours après la première dose de nivolumab ${ }^{7}$. Ce cas est intéressant, puisque le patient prenait d'autres médicaments qui pouvaient être associés à ce type de réaction, soit de l'allopurinol, le triméthoprimesulfaméthoxazole et le fluconazole ${ }^{7}$. Finalement, un cas similaire à celui de notre patiente concerne un homme de 57 ans qui a développé une NET seulement six jours après la première administration d'ipilimumab et de nivolumab pour traiter un adénocarcinome gastro-œsophagien ${ }^{8}$.

Environ 37 à $70 \%$ des patients traités avec l'ipilimumab développent une toxicité cutanée, dont 1 à $3 \%$ de grade
3 ou supérieur. De façon générale, 30 à $50 \%$ des patients sous immunothérapie développent une toxicité cutanée durant leur traitement ${ }^{2}$. La monographie de l'ipilimumab ${ }^{9}$ indique que le délai médian d'apparition d'une réaction cutanée à médiation immunitaire est de 3,1 semaines, ce qui correspond aussi au délai d'action du médicament sur les lymphocytes T. La monographie du nivolumab ${ }^{10}$ indique que le délai médian d'apparition de réactions cutanées de grade 3 à médiation immunitaire lorsqu'il est combiné à l'ipilimumab pour le traitement d'un mélanome est de deux semaines et que la fréquence de telles réactions est plus élevée lorsqu'il est utilisé en combinaison.

Bien que le mécanisme sous-jacent à la NET induite par l'immunothérapie ne soit pas clairement élucidé, plusieurs hypothèses ont été émises. D’abord, l'augmentation de l'activité antitumorale des lymphocytes $\mathrm{T}$ pourrait être le déclencheur de réactions auto-immunes contre les kératinocytes. Ensuite, les anti PD-1 peuvent compromettre l'intégrité de l'épithélium, mais il est difficile de déterminer si ce phénomène peut contribuer au développement d'une $\mathrm{NET}^{11}$. Certains auteurs ont envisagé la possibilité que la comédication augmente les risques de développer une $\mathrm{NET}^{10}$. L'immunothérapie pourrait être le déclencheur d'une réaction d'hypersensibilité cutanée favorisée par la prise de médicaments connus pour en augmenter le risque.

Concernant les autres médicaments pris par la patiente, la mention du SSJ apparaît dans quelques monographies de produits, comme le dexlansoprazole ${ }^{12}$, le tramadolacétaminophène ${ }^{13}$ et la saxagliptine ${ }^{14}$, mais l'incidence n'est pas précisée. Concernant le lévétiracétam, l’incidence de SSJ serait supérieure à celle de la population générale et surviendrait en moyenne après 12 jours ${ }^{15}$. Toutefois, la patiente prenait ces médicaments depuis plus longtemps et aucun n’a été retiré durant le traitement. Concernant l'amlodipine, il y a moins de $0,1 \%$ de cas de SSJ selon la monographie ${ }^{16}$ et la littérature scientifique ne décrit que peu de cas portant sur cet agent. Dans le cas présent, l'amlodipine a d'abord été suspectée comme cause potentielle de la NET, puisque son administration avait débuté deux semaines avant la réaction. Mais cette hypothèse a été écartée, puisque le médicament a été repris pendant l'hospitalisation sans aggravation de la réaction. Il faut également prendre en considération le triméthoprime-sulfaméthoxazole que la patiente venait de commencer à prendre. Bien que le traitement se soit terminé 14 jours avant la réaction, le risque de SSJ est connu avec cet antibiotique $^{17}$. Il est donc raisonnable d'affirmer quaucun de ces médicaments nétait directement en cause dans ce cas-ci, mais on ne peut exclure leur implication une fois l'inhibition lymphocytaire levée.

La revue de littérature confirme que des réactions de SSJ et de NET sont possibles avec la prise d'ipilimumab et de nivolumab, ce qui renforce la thèse postulant que cette patiente a réagi à cette double immunothérapie. L'intérêt du cas vient du fait que la réaction est survenue seulement 
trois jours après l'exposition à ces agents, alors que des délais de 7 à 140 jours sont mentionnés dans les autres rapports de $\mathrm{cas}^{18}$. Selon plusieurs références, le délai d'apparition d'un SSJ ou d'une NET varierait d'une à quatre semaines après le début du traitement médicamenteux, mais pourrait aller jusqu'à huit semaines ${ }^{19,20}$. Devant l'incertitude à propos des rôles respectifs de l'immunothérapie et des autres médicaments augmentant le risque de réaction d'hypersensibilité cutanée, il conviendrait de prendre en considération ce risque de réaction sévère dans lévaluation globale précédant la prescription d'une immunothérapie.

En conclusion, ce cas illustre la survenue d'une NET chez une femme de 58 ans traitée pour un mélanome métastatique avec la combinaison d'ipilimumab et de nivolumab. La rapidité de l'apparition de la réaction après le début de l'immunothérapie est surprenante lorsqu'on la compare avec ce que rapporte la littérature disponible.

\section{Références}

1. Hassel JC, Heinzerling L, Aberle J, Bähr O, Eigentler TK, Grimm $\mathrm{MO}$, et al. Combined immune checkpoint blockade (anti-PD-1/antiCTLA-4): evaluation and management of adverse drug reactions. Cancer Treat Rev. 2017(57):36-49.

2. Brahmer JR, Lacchetti C, Schneider BJ, Atkins MB, Brassil KJ, Caterino JM, et al. Management of immune-related adverse events in patients treated with immune checkpoint inhibitor therapy: American Society of Clinical Oncology clinical practice guideline. J Clin Oncol. 2018;36(17):1714-68.

3. Cohen V. Toxic epidermal necrolysis (TEN). Medscape; le 20 décembre 2018. Consulté sur intranet, Université de Montréal.

4. Naranjo CA, Busto U, Sellers EM, Sandor P, Ruiz I, Roberts EA, et al. A method for estimating the probability of adverse drug reactions. Clin Pharmacol Ther. 1981;30(2):239-45.

5. Nayar N, Briscoe K, Fernandez Penas P. Toxic epidermal necrolysislike reaction with severe satellite cell necrosis associated with nivolumab in a patient with ipilimumab refractory metastatic melanoma. J Immunother. 2016;39(3):149-52.

6. Logan IT, Zaman S, Hussein L, Perrett CM. Combination therapy of ipilimumab and nivolumab-associated toxic epidermal necrolysis (TEN) in a patient with metastatic melanoma: a case report and literature review. J Immunother. 2020;43(3):89-92.

7. Griffin LL, Cove-Smith L, Alachkar H, Radford JA, Brooke R, Linton KM. Toxic epidermal necrolysis (TEN) associated with the use of nivolumab (PD-1 inhibitor) for lymphoma. JAAD Case Rep. 2018;4(3):229-31.

8. Kubicki SL, Welborn ME, Patel AB. Toxic epidermal necrolysis during cotherapy with ipilimumab and nivolumab. J Immunother Precis Oncol. 2018;1(2):78-81.

9. Yervoy product monograph. Bristol-Myers Squibb Canada; le 13 janvier 2020.

10. Opdivo product monograph. Bristol-Myers Squibb Canada; le 30 octobre 2019.
11. Fay AP, Brandão Moreira P, Nunes Filho PRS, Albuquerque C, Barrios $\mathrm{CH}$. The management of immune-related adverse events associated with immune checkpoint blockade. Expert Rev Qual Life Cancer Care. 2016:1(1) :89-97.

12. Dexilant product monograph. Takeda Canada Inc; le 6 janvier 2020.

13. Auro-tramadol/acetaminophen product monograph. Auro Pharm Inc; le 28 juin 2018.

14. Onglyza product monograph. AstraZeneca Canada Inc; le 12 décembre 2018.

15. Levetiracetam product monograph. Sivem Produits Pharmaceutiques ULC; le 17 juin 2017.

16. GD-amlodipine product monograph. Pfizer Canada Inc; le 23 octobre 2017.

17. Sulfatrim product monograph. AA Pharma Inc; le 17 janvier 2019.

18. Chen CB, Wu MY, Ng CY, Lu CW, Wu J, Kao PH, et al. Severe cutaneous adverse reactions induced by targeted anticancer therapies and immunotherapies. Cancer Manag Res. 2018;10:1259-73.

19. Sassolas B, Haddad C, Mockenhaupt M, Dunant A, Liss Y, Bork K, et al. ALDEN, an algorithm for assessment of drug causality in StevensJohnson syndrome and toxic epidermal necrolysis: comparison with case-control analysis. Clin Pharmacol Ther. 2010;88(1):60-8.

20. Law RM, Law DTS. Dermatologic drug reactions and common skin conditions. Dans : DiPiro JT, Talbert RL, Yee GC, Matzke GR, Wells BG, Posey LM, rédacteurs. Pharmacotherapy: a pathophysiologic approach. 10 édition. McGraw-Hill Education; 2017.

Marika Lepage-Légaré, Pharm. D., était, lors de l'écriture de cette article, résidente en pharmacie au Centre intégré universitaire de santé et de services sociaux (CIUSSS) de l'Estrie, CHU de Sherbrooke, Sherbrooke (Québec). Elle est actuellement pharmacienne au même organisation.

Sandrine Léger, Pharm. D., était, lors de l'écriture de cette article, résidente en pharmacie au Centre intégré de santé et de services sociaux (CISSS) de Laval, Hôpital de la Cité-de-la-Santé, Laval (Québec). Elle est actuellement pharmacienne au même organisation.

Hugo Ricignuolo, Pharm. D., était, lors de l'écriture de cette article, résident en pharmacie au Centre intégré de santé et de services sociaux (CISSS) de Laval, Hôpital de la Cité-de-la-Santé, Laval (Québec). Il est actuellement pharmacien au CISSS de la Côte-Nord, Baie-Comeau (Québec).

Gabrielle St-Louis, Pharm. D., était, lors de l'écriture de cette article, résidente en pharmacie au Centre intégré de santé et de services sociaux (CISSS) de Laval, Hôpital de la Cité-de-la-Santé, Laval (Québec). Elle est actuellement pharmacienne au même organisation.

Thomas Joly-Mischlich, B. Pharm., M. Sc., est pharmacien au Centre intégré universitaire de santé et de services sociaux (CIUSSS) de l'Estrie, CHU de Sherbrooke, Sherbrooke (Québec).

Conflits d'intérêts : Aucune déclaration.

Adresse de correspondance :

Thomas Joly-Mischlich

Centre intégré universitaire de santé et des services sociaux de l'Estrie

$\mathrm{CHU}$ de Sherbrooke

$3001,12^{\mathrm{e}}$ avenue nord

Sherbrooke QC J1H 5H3

Courriel : thomas.joly-mischlich.ciussse-chus@ssss.gouv.qc.ca

Financement : Aucune reçue. 